\title{
Application of positron annihilation lifetime spectroscopy to nano-characterisation of polymer-modified mortars
}

\author{
P. Guagliardo ${ }^{1}$, A. J. Klemm ${ }^{2}$, S. N. Samarin ${ }^{1}$ \& J. F. Williams ${ }^{1}$ \\ ${ }^{1}$ ARC Centre of Excellence for Antimatter-Matter Studies, \\ School of Physics, University of Western Australia, Australia \\ ${ }^{2}$ School of Built and Natural Environment, \\ Glasgow Caledonian University, UK
}

\begin{abstract}
Positron annihilation lifetime spectroscopy (PALS) has been applied to study the microstructural features of immature cement mortars. Two types of cement mortars containing superabsorbent polymers (SAPs) were studied, in addition to Ordinary Portland Cement (OPC). The ortho-positronium lifetimes for all samples were in the range of 1.70-1.73 ns, values that are close to that of free water $(1.7 \mathrm{~ns})$ and hence suggest the presence of water-filled pores. Periodic lifetime measurements showed that the intensity of this component decreased slightly over a period of four weeks, indicating water loss associated with the curing process, evaporation or a combination of the two.

Keywords: cement mortar, superabsorbent polymer, positron annihilation lifetime spectroscopy, positronium, porosity, hydration, curing.

\section{Introduction}

Cement is a material of immense practical importance, but in spite of its almost ubiquitous use, its microstructural characterisation still proves to be problematic. The complexity of the problem is enhanced when the composition of cement is modified through the use of auxiliary agents. Ordinary Portland cement is a combination of gypsum $\left(\mathrm{CS}^{*} \mathrm{H}_{2}\right)$ and clinker. Gypsum acts to prevent rapid setting of the cement paste while clinker is the hydraulic binder. The major constituents of clinker are tricalcium silicate $\left(\mathrm{C}_{3} \mathrm{~S}\right)$, dicalcium silicate $\left(\mathrm{C}_{2} \mathrm{~S}\right)$,
\end{abstract}


tricalcium aluminate and tetracalcium alumina-ferrite $\left(\mathrm{C}_{4} \mathrm{AF}\right) ; \mathrm{C}=\mathrm{CaO}, \mathrm{S}=$ $\mathrm{SiO}_{2}, \mathrm{~A}=\mathrm{Al}_{2} \mathrm{O}_{3}, \mathrm{~F}=\mathrm{Fe}_{2} \mathrm{O}_{3}, \mathrm{~S}^{*}=\mathrm{SO}_{3}$ and $\mathrm{H}=\mathrm{H}_{2} \mathrm{O}$ (abbreviations commonly used in industrial nomenclature) $[1,2]$.

The presence of water among the active compounds of cement leads to a rearrangement of their structure and the initiation of the hardening process. The initial hardening is caused by the hydration of $\mathrm{C}_{3} \mathrm{~S}$, which forms a gel-like silicate and calcium hydrate phase referred to as C-S-H gel. These particles ultimately crystallise and bind together the particles of sand or stone into a hard mass. Other hydrates of the complexes described above are also formed, although the reaction rates may differ considerably. The final product is a hardened cement paste which is a mixture of unreacted cement particles, hydration products and pores. Two types of pores are usually distinguished capillary pores, which comprise the water- or air-filled spaces between the hydrates, with sizes in the range of $10-1000 \mathrm{~nm}$, and smaller pores referred to as gel pores, which are contained in the amorphous hydrate phase. Gel and capillary pores form a continuous network of pores throughout the material $[3,4]$.

The mechanical properties of cementitious materials are heavily influenced by porosity, with the volume and size distribution of pores controlling both the strength and durability of the material [5]. A detailed characterisation of the pore structure and the factors that affect it are thus crucial to advancing the design of these materials; however, classical porosimetry methods such as mercury intrusion porosimetry (MIP) and gas adsorption cannot always be relied upon to extract information on pores smaller than a few nanometres [6]. In this regard, positron annihilation lifetime spectroscopy (PALS) has considerable advantages over these classical methods. PALS is sensitive to both open and closed porosity, for example, whereas isolated pores are invisible to the aforementioned techniques. In addition, PALS is sensitive to pores in the size range of $0.3-30 \mathrm{~nm}$ (that is, in the size range of gel pores), and both pore sizes and relative concentrations can be measured (refer to the following section for a detailed explanation) [7]. In this work, PALS was used to study the features of the porosity of three cement mortars. Two types of cement mortars containing superabsorbent polymers (SAPs) were studied, in addition to a sample composed only of OPC.

SAPs are cross-linked networks of hydrophilic polymers with a high capacity for water uptake - they can absorb and retain up to 500 times their weight in water. This makes them ideal for use in water-absorbing applications such as absorbent medical dressings and controlled release media [8]. During the mixing process the polymers absorb the pore solution immediately after their addition to the mortar, reaching saturation within minutes $[9,10]$. They then swell to form spherical cavities filled with water. At later stages of the hydration process the water is released to the concrete matrix and the cavities remain as empty pores. For mortars with a low water-to-cement ratio, it is possible to replace part of the irregular capillary pores with larger spherical pores formed by saturated polymers. The dispersion and size of these pores can be estimated by the material attributes of polymers. 
The samples were studied with PALS over a period of 4 weeks with measurements starting when the samples were aged 21 days, in order to elucidate the changes in nano-porosity caused by the addition of SAPs as well as the changes arising from the curing/hydration process. In addition, samples aged at 3 and 6 weeks were studied with MIP to determine changes in their pore distributions and bulk densities. MIP can provide information on larger pores (from $100 \mathrm{~nm}$ to $100 \mu \mathrm{m}$ ) to which PALS is not sensitive.

\section{Positron annihilation background}

Positron annihilation techniques have been applied to study nano-porosity in a wide range of materials, such as zeolite, silicates and polymers [7]. In positron annihilation lifetime spectroscopy (PALS), positrons from a ${ }^{22} \mathrm{Na}$ radioisotope are injected into the sample under study and a positron lifetime spectrum is recorded [11]. As a $1.274 \mathrm{MeV}$ gamma-quantum is emitted during the decay of ${ }^{22} \mathrm{Na}$ almost simultaneously with a positron, the lifetime is defined as the time between detection of the $1.274 \mathrm{MeV}$ photon and the subsequent $511 \mathrm{keV}$ photons created from the positron's annihilation with an electron. The measured spectrum is a histogram of the time periods between these two events. Ideally, it is a sum of decaying exponentials of the form

$$
\sum \frac{I_{j}}{\tau_{j}} e^{-t / \tau_{j}}
$$

where $I_{j}$ is the intensity or weighting of the $j^{\text {th }}$ positron state with lifetime $\tau_{j}$. An experimentally obtained spectrum differs from this form however, in that it is convolved with the time response function of the apparatus, usually approximated by a sum of Gaussians.

Porosimetry is made possible by exploiting the phenomenon of positronium (Ps) formation, Ps being the hydrogen-like bound state of a positron and an electron [12]. In brief, positrons penetrate the material under investigation and rapidly lose energy, predominately through ionising collisions. Ps formation can then take place as a reaction between the positron and a secondary electron produced during the positrons' thermalisation (these secondary electrons are often termed spur electrons [13]). Positronium is formed in two spin states with dramatically different annihilation characteristics. The singlet state (with antiparallel orientation of spins) is termed para-positronium (p-Ps); it has a vacuum lifetime of $125 \mathrm{ps}$ and decays via the emission of two gamma quanta. The triplet state (with parallel spins of electron and positron) is referred to as orthopositronium (o-Ps) and has a much longer vacuum lifetime of $142 \mathrm{~ns}$, decaying via three gamma emission. Due to its short lifetime, p-Ps is not significantly perturbed by the material; however, o-Ps, because of its intrinsically longer lifetime, interacts strongly with the material and its pore structure. In the presence of matter, o-Ps can decay into two gammas via a process known as pick-off annihilation - the positron in o-Ps annihilates with an electron of opposite spin in the material via a two-gamma process. As a result of pick-off 
annihilation, the o-Ps lifetime in matter is significantly shorter than its vacuum value (and can be as low as $\sim 1 \mathrm{~ns}$ ) [7].

The basis for studying porosity is that o-Ps tends to localise in regions of low density, such as pores. This is because in the bulk of the material the positron and the electron in o-Ps experience repulsive Coulomb and exchange forces respectively. As a result, most of these materials (porous insulators) have a negative work function for positronium [7]. Porosimetry is possible because the annihilation rate (the inverse of the lifetime) in the pore is a function of the pore size - smaller pores have a shorter lifetime compared to large pores, where the overlap of the Ps wave function with the pore walls is reduced. A semi-empirical quantum mechanical relation has been developed by Tao [14] and Eldrup et al. [15] to relate the o-Ps lifetimes to pore radii:

$$
\frac{1}{\tau}=2\left[1-\frac{\mathrm{R}}{\mathrm{R}+\Delta \mathrm{R}}+\frac{1}{2 \pi} \sin \left(\frac{2 \pi \mathrm{R}}{\mathrm{R}+\Delta \mathrm{R}}\right)\right]
$$

where $\tau$ is the lifetime, $\mathrm{R}+\Delta \mathrm{R}$ is the pore radius and $\Delta \mathrm{R}$ is empirically determined to be $0.16-0.17 \mathrm{~nm}$. This model is applicable to sub-nanometre pores of a spherical geometry. Gidley et al. [16, 17] extended this model to include larger pore sizes and varying pore geometries (cylinders, cubes, channels and sheets).

\section{Experimental}

\subsection{Materials and mixes}

For the purpose of this research Portland cement (BS EN 197-1 CEM II/B-V 32,5) was mixed at 1:1 ratio with fine sand (the vast majority of particles were distributed below $0.6 \mathrm{~mm}$ ). Throughout the investigation the total water-tocement ratio of 0.45 was maintained. The mix compositions are presented in Table 1. The pastes were shaped into cylinders with diameters of about $25 \mathrm{~mm}$ and thicknesses in the range of 5-10 $\mathrm{mm}$.

Table 1: $\quad$ Composition of cement mortars.

\begin{tabular}{|c|c|c|c|}
\hline Sample designation & OPC & SAP-A & SAP-B \\
\hline Mix code & $\mathrm{R}$ & $\mathrm{A}$ & $\mathrm{B}$ \\
\hline (Water/Cement) $_{\text {total }}$ & 0.45 & 0.45 & 0.45 \\
\hline (Water/Cement) $_{\text {effective }}$ & 0.45 & 0.425 & 0.438 \\
\hline Sand/Cement & 1 & 1 & 1 \\
\hline SAP content [\%] (by cement weight) & 0 & 0.25 & 0.25 \\
\hline
\end{tabular}


The SAPs used in this study were cross-linked polymers provided by BASF. SAP-A is a copolymer of acrylamide and acrylic acid and SAP-B is a polymer based on acrylic acid. The products had absorption capacities of $200-250 \mathrm{ml} / \mathrm{g}$ in demineralised water, though the absorption in mortar depended on the product and was approximately $10 \mathrm{~mL} / \mathrm{g}$ for SAP-A and $5 \mathrm{~mL} / \mathrm{g}$ for SAP-B. Both materials were prepared by grinding and screening to sizes of $63-125 \mu \mathrm{m}$, but there was also a minor (less than 10\%) content of finer particles. The SAP particle shapes were irregular and their sizes after initial absorption were in the range of $135-270 \mu \mathrm{m}$ for SAP-A and 105-210 $\mu \mathrm{m}$ for SAP-B.

\subsection{Positron lifetime and mercury intrusion measurements}

For positron lifetime measurements, approximately $30 \mu \mathrm{Ci}$ of ${ }^{22} \mathrm{NaCl}$ was deposited on $7 \mu \mathrm{m}$ Kapton foil and covered with an identical foil. The edges of this foil-sandwich were then sealed, and this source foil was placed between two identical pieces of sample. The positron lifetimes were measured with a fast-fast coincidence system. The gamma-ray detectors consist of a truncated cone (31.8 $\mathrm{mm}$ diameter tapering to $12.7 \mathrm{~mm}$ with a height of $12.7 \mathrm{~mm}$ ) BC418 scintillator coupled to a Burle 8850 photomultiplier tube. The time resolution of the system is approximately $220 \mathrm{ps}$, as determined from analysis of a spectrum of highpurity annealed nickel. The spectra comprise of at least 2 million counts and have been analysed using PAScual version 1.3.0 [18].

Mercury intrusion porosimetry (MIP) has been carried out with the use of a Porosimeter Autopore IV 9500 by Micromeritics, with a pressure range up to $60000 \mathrm{psi}$ for all samples at ages 3 and 6 weeks.

\section{Results and discussion}

In Table 2 the fitted lifetimes and intensities for all samples are given at four different ages. In each case, the lifetime spectra could be fitted with three discrete components. In porous media a number of annihilation processes are possible because multiple positron and positronium states exist. It is likely that the two shorter lifetime components $\left(\tau_{1}\right.$ and $\left.\tau_{2}\right)$ in Table 2 are the averages of several different annihilation modes. These could include positron annihilation (of both free and trapped positrons), p-Ps self-annihilation and o-Ps pick-off annihilation in the bulk of the material, and annihilations in the source foil.

Nanosecond lifetime components $\left(\tau_{3}\right)$ on the other hand are typically associated with o-Ps pick-off annihilation in nano-pores. For all of the cement samples the average $\tau_{3}$ over the course of the measurements was in the range of 1.70-1.73 ns. The presence of a nanosecond component is often an indicator of porosity or free volume, and these values would correspond to pores having diameters in the range of 0.51-0.52 $\mathrm{nm}$ (using the TE model, equation 2). However, these lifetimes are very close to that observed in pure water, approximately $1.7 \mathrm{~ns}$ [19]. Given that cement pastes are known to contain a significant fraction of water, and that the samples were tested at a relatively young age (testing began at 21 days after mixing), it seems likely that these 
nanosecond components are associated with annihilation in water contained in capillary pores of the cement and hence cannot be directly related to a pore size. Consolati and Quasso [20, 21], Consolati et al. [22] and Salgueiro et al. [23] observed similar lifetimes in Portland cement pastes and attributed this component to water-filled pores.

Table 2: $\quad$ Lifetimes and intensities; standard deviations are given in brackets.

\begin{tabular}{|c|c|c|c|c|c|c|c|c|}
\hline & $\begin{array}{c}\text { Age } \\
\text { (weeks) }\end{array}$ & $\tau_{1}(\mathrm{ps})$ & $\tau_{2}(\mathrm{ps})$ & $\tau_{3}(\mathrm{~ns})$ & $I_{1}(\%)$ & $I_{2}(\%)$ & $I_{3}(\%)$ & $\chi^{2}$ \\
\hline \multirow{4}{*}{$\sum_{\infty}^{\mathbb{1}}$} & 3 & $218(9)$ & $410(10)$ & $1.67(0.02)$ & $43(4)$ & $50(3)$ & $5.9(0.1)$ & 0.93 \\
\hline & 4 & $227(9)$ & $410(10)$ & $1.67(0.03)$ & $46(5)$ & $48(5)$ & $5.7(0.2)$ & 1.04 \\
\hline & 5 & $242(7)$ & $430(10)$ & $1.73(0.03)$ & $55(4)$ & $40(4)$ & $5.2(0.2)$ & 0.99 \\
\hline & 6 & $233(8)$ & $420(10)$ & $1.72(0.03)$ & $50(4)$ & $44(4)$ & $5.2(0.2)$ & 0.99 \\
\hline \multirow{4}{*}{$\sum_{\infty}^{\infty}$} & 3 & $219(5)$ & $409(7)$ & $1.73(0.02)$ & $45(2)$ & $49(2)$ & $5.0(0.1)$ & 1.15 \\
\hline & 4 & $229(5)$ & $421(9)$ & $1.76(0.03)$ & $49(3)$ & $46(3)$ & $4.0(0.1)$ & 1.08 \\
\hline & 5 & $221(6)$ & $409(8)$ & $1.71(0.03)$ & $46(3)$ & $50(3)$ & $4.3(0.1)$ & 1.04 \\
\hline & 6 & $237(6)$ & $420(10)$ & $1.63(0.03)$ & $53(4)$ & $43(4)$ & $4.2(0.2)$ & 1.08 \\
\hline \multirow{4}{*}{ อิ } & 3 & $224(6)$ & $402(8)$ & $1.71(0.02)$ & $47(3)$ & $48(3)$ & $4.6(0.1)$ & 1.01 \\
\hline & 4 & $239(5)$ & $430(10$ & $1.67(0.02)$ & $57(3)$ & $39(2)$ & $4.3(0.1)$ & 1.01 \\
\hline & 5 & $238(5)$ & $420(10)$ & $1.67(0.03)$ & $53(3)$ & $42(3)$ & $4.2(0.1)$ & 1.09 \\
\hline & 6 & $212(9)$ & $390(10)$ & $1.73(0.03)$ & $43(3)$ & $53(4)$ & $4.3(0.1)$ & 1.07 \\
\hline
\end{tabular}

In Figure 1, o-Ps lifetimes $\left(\tau_{3}\right)$ and intensities $\left(\mathrm{I}_{3}\right)$ are plotted as a function of sample age. Lifetime spectra were recorded every 7 days for a 4 week period. Over the course of the measurements, $\mathrm{I}_{3}$ for the cement sample containing SAP$\mathrm{A}$ is somewhat higher than for the cement containing SAP-B and the pure cement sample. If these lifetimes are indicative of water-filled pores then this suggests a higher concentration of pores in the sample containing SAP-A. As the results for SAP-B are closer to those of pure cement, this suggests that the presence of the polymer does not significantly alter the pore concentration.

For the SAP-A sample, a gradual decrease in $\mathrm{I}_{3}$ is observed over weeks 3-5, whereas for the SAP-B and pure cement samples there is a decrease after the initial measurement (weeks 3-4), and then $\mathrm{I}_{3}$ is relatively constant. While these changes are small, they may be indicative of various stages of the hydration process, as the decrease in $\mathrm{I}_{3}$ is consistent with the reduction in total porosity that is known to occur as hydration progresses [3]. The gradual decline seen in the SAP-A sample suggests that this polymer releases its water more slowly compared to SAP-B.

At the onset of the measurement (age 21 days) the hydration process is likely to be still underway, with both di- and tri-calcium silicates reacting with water to produce a network of calcium silicate hydrates. Water could then be consumed in the hydration process for at least the next two weeks. However, during this time it is difficult to say if the loss of water seen by the decrease in $I_{3}$ is also associated with evaporation. As the samples lose water one might expect the lifetimes to increase as there would be a transition from filled to empty pores. 
The fact that this does not occur suggests that pores collapse with water loss (the volume of capillary pores is known to decrease with hydration [3]).

These results are consistent with the work of Consolati and Quasso [20, 21] who observed a systematic decrease in $\mathrm{I}_{3}$ with sample age, but they are in contrast to the work of Myllylä and Karras [24] where the opposite trend was seen. It should be noted however that a direct comparison with these studies is not possible due to differing experimental conditions (namely the composition and age of samples).
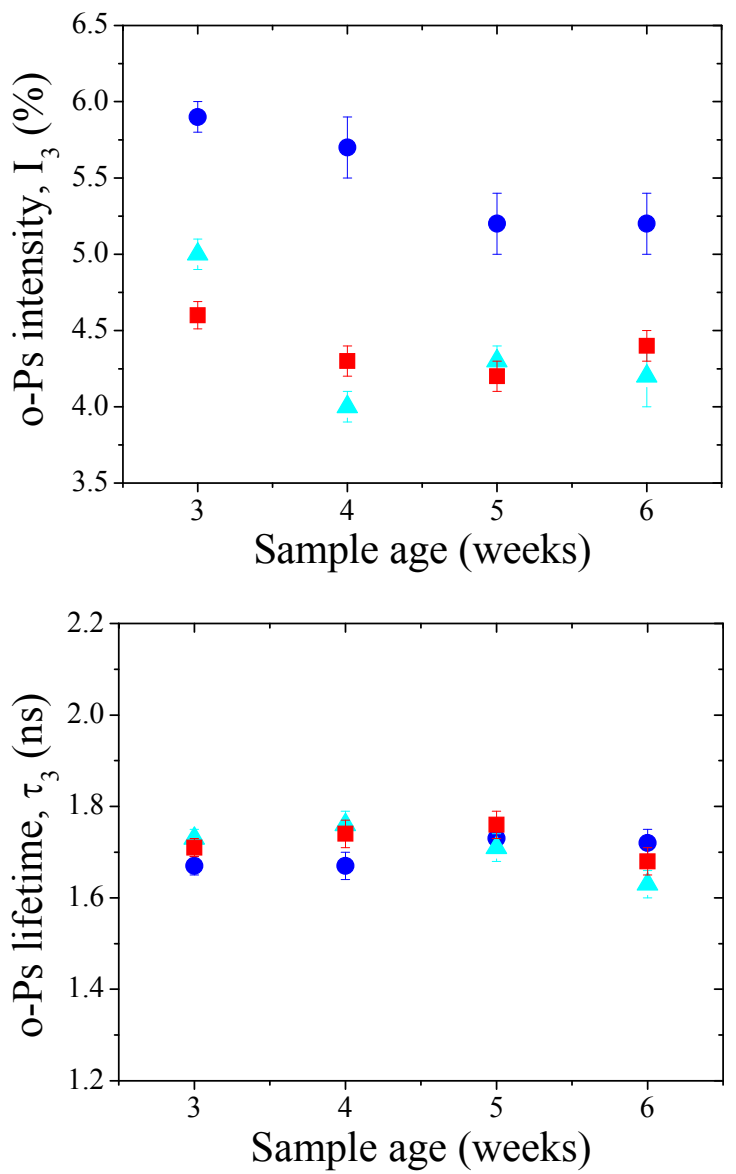

Figure 1: o-Ps intensities (top) and lifetimes (bottom) vs. sample age for SAP-A (circles), SAP-B (triangles) and Ordinary Portland Cement (squares).

In addition to PALS, MIP measurements were carried out on samples aged 3 and 6 weeks; the resulting pore distributions are shown in Figure 2. It is apparent that the samples contain a wide distribution of pores, with the majority of pores 
in the size range of 10-100 nm. The MIP results are in qualitative agreement with the PALS data in the sense that the sample containing SAP-B displays similar behaviour to pure cement - at 6 weeks their pore distributions almost coincide and the total porosity for both samples has decreased with aging. This decrease is also consistent with the reduction in $\mathrm{I}_{3}$ seen in the PALS data.

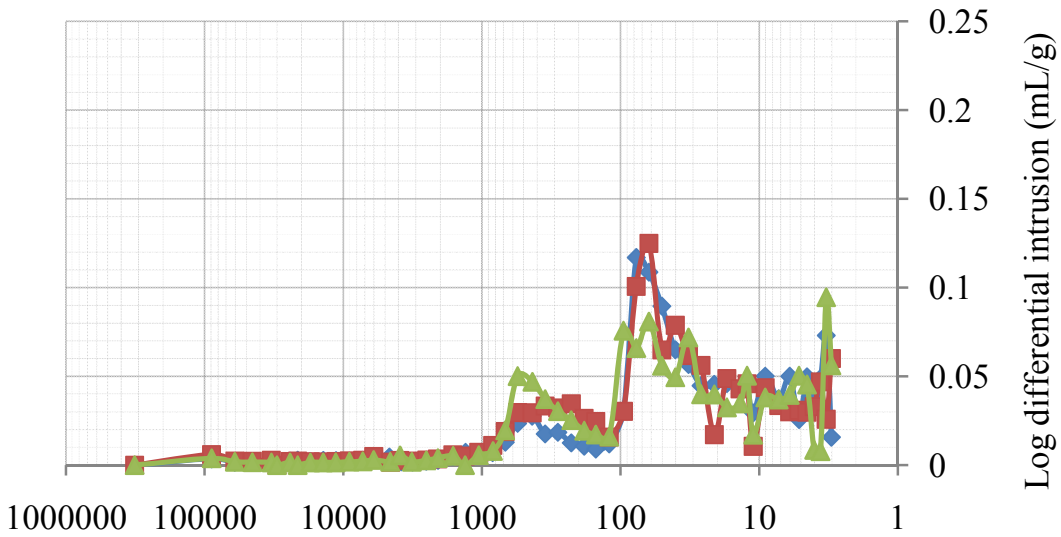

Pore size diameter (nm)

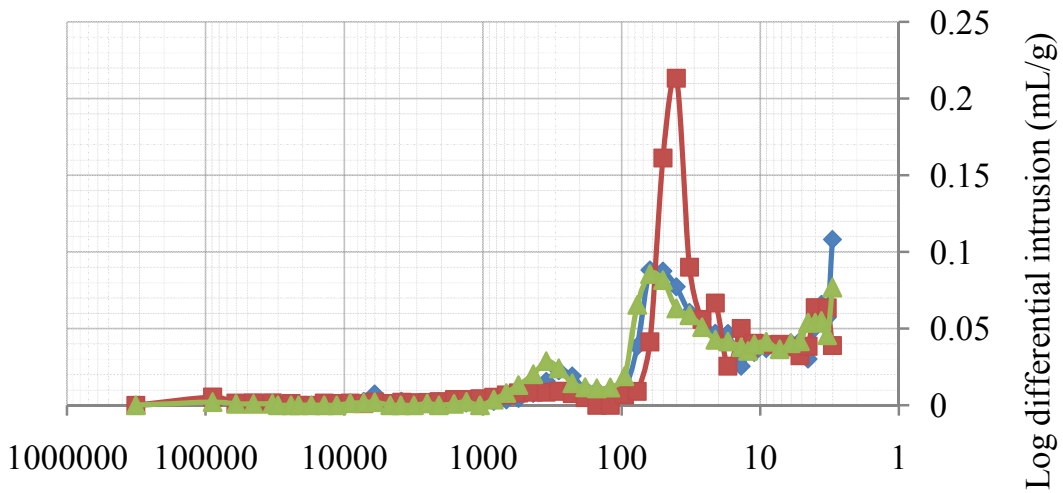

Pore size diameter $(\mathrm{nm})$

Figure 2: $\quad$ Pore size distributions determined by MIP at age 3 weeks (top) and 6 weeks (bottom); OPC - diamonds, SAP-A - squares, SAP-B triangles.

Conversely, the SAP-A sample shows a significant increase in the pore concentration within the 10-100 nm range at 6 weeks. This is in contrast to the decrease in $\mathrm{I}_{3}$ seen in PALS, although PALS may not be sensitive to the porosity in this size range. In spite of the differing trend, both PALS and MIP show that 
the presence of SAP-A significantly alters the hydration process of the cement mortar.

The bulk densities of the samples were also determined by MIP measurements (Table 3). It was found that the density of all samples increased with aging time. Again, the changes in density for the pure cement and SAP-B samples are similar. The change in density for SAP-A is much smaller, which is consistent with the slower rate of change of $\mathrm{I}_{3}$.

Table 3: $\quad$ Bulk densities determined by MIP at 3 and 6 weeks.

\begin{tabular}{|c|c|c|}
\hline Sample & $\begin{array}{c}\text { Bulk density at 3 weeks } \\
(\mathbf{g} / \mathbf{m L})\end{array}$ & $\begin{array}{c}\text { Bulk density at 6 weeks } \\
(\mathbf{g} / \mathbf{m L})\end{array}$ \\
\hline OPC & 1.83 & 1.89 \\
\hline SAP-A & 1.82 & 1.86 \\
\hline SAP-B & 1.84 & 1.91 \\
\hline
\end{tabular}

In order to gain further insight into the nature of the porosity, the samples were heated at $116^{\circ} \mathrm{C}$ for 4 hours under vacuum conditions $\left(10^{-5}\right.$ Torr) and lifetime measurements were repeated. Lifetime spectra were recorded directly after heating and the samples were sealed in a plastic bag containing a desiccant (to prevent water absorption) during the measurement. The resulting lifetimes and intensities are given in Table 4 and the corresponding pore sizes are given in Table 5.

At least three types of water can be distinguished in a hydrating cement paste. Chemically-bound water is directly incorporated into the structure of the hydration products, physically-bound water is absorbed on the surfaces of cement particles and reaction products, and there is also "free" water contained in the capillary and gel pores [25]. The heat treatment will have removed a significant fraction of the latter two sources of water; however, much higher temperatures (above $950^{\circ} \mathrm{C}$ ) are required to remove chemically-bound water.

Table 4: $\quad$ Lifetimes and intensities obtained after heat treatment.

\begin{tabular}{|c|c|c|c|}
\cline { 2 - 4 } \multicolumn{1}{c|}{} & \multicolumn{3}{c|}{ Sample } \\
\cline { 2 - 4 } \multicolumn{1}{c|}{} & OPC & SAP-A & SAP-B \\
\hline$\tau_{\mathbf{1}} \mathbf{( p s )}$ & $263(2)$ & $214(9)$ & $219(4)$ \\
\hline$\tau_{\mathbf{2}} \mathbf{( p s )}$ & $536(7)$ & $380(9)$ & $413(5)$ \\
\hline$\tau_{\mathbf{3}} \mathbf{( n s )}$ & $4.3(0.1)$ & $1.32(0.07)$ & $1.62(0.05)$ \\
\hline$\tau_{\mathbf{4}} \mathbf{( n s )}$ & - & $12.6(0.7)$ & $14.9(0.6)$ \\
\hline $\mathbf{I}_{\mathbf{1}} \mathbf{( \% )}$ & $75(2)$ & $36(8)$ & $44(2)$ \\
\hline $\mathbf{I}_{\mathbf{2}} \mathbf{( \% )}$ & $24(1)$ & $60(7)$ & $52(2)$ \\
\hline $\mathbf{I}_{\mathbf{3}} \mathbf{( \% )}$ & $1.3(0.1)$ & $3.3(0.4)$ & $2.5(0.1)$ \\
\hline $\mathbf{I}_{\mathbf{4}} \mathbf{( \% )}$ & - & $1.0(0.1)$ & $0.70(0.01)$ \\
\hline $\boldsymbol{\chi} \mathbf{2}$ & 1.13 & 1.05 & 1.01 \\
\hline
\end{tabular}


After heat treatment, $\tau_{3}$ for OPC increases to $4.3 \mathrm{~ns}$. This corresponds to a gel pore diameter of $0.86 \mathrm{~nm}$ using the TE model (equation 2). However, the intensity of this component decreases by a factor of about 3.3; this could suggest that a significant fraction of the pores collapse upon water removal. There is also a significant redistribution of intensity from $\mathrm{I}_{2}$ to $\mathrm{I}_{1}$ after heat treatment. These are the shorter components associated with multiple positron states in the material. The redistribution of intensity from the longer component $\left(\tau_{2}\right)$ to the shorter one $\left(\tau_{1}\right)$ indicates that the heat treatment has reduced the number of positron traps in the bulk material. This could be associated with an increase in the bulk density after heating.

Table 5: $\quad$ Pore diameters, $\mathrm{d}_{3}$ and $\mathrm{d}_{4}$, calculated from o-Ps lifetimes, and total o-Ps intensities $\left(\mathrm{I}_{\mathrm{o}-\mathrm{Ps}}\right)$.

\begin{tabular}{|c|c|c|c|}
\cline { 2 - 4 } \multicolumn{1}{c|}{} & \multicolumn{3}{c|}{ Sample } \\
\cline { 2 - 4 } \multicolumn{1}{c|}{} & OPC & SAP-A & SAP-B \\
\hline $\mathbf{d}_{\mathbf{3}} \mathbf{( n m )}$ & 0.86 & 0.42 & 0.50 \\
\hline $\mathbf{d}_{\mathbf{4}} \mathbf{( n m )}$ & - & 1.45 & 1.56 \\
\hline $\mathbf{I}_{\mathbf{0}-\mathbf{P}}$ before (\%) & 4.3 & 5.2 & 4.2 \\
\hline $\mathbf{I}_{\mathbf{0}-\mathbf{P s}}$ after (\%) & 1.3 & 4.3 & 3.2 \\
\hline
\end{tabular}

For the cement samples containing SAPs an additional low intensity, long lifetime component $\left(\tau_{4}\right)$ appears in the spectra after heat treatment (and due to the presence of this long component it was necessary to fix the background to the left of the main coincidence peak to the average value in the analysis). $\tau_{4}$ may be associated with pores formed by the polymers which have replaced some of the ordinary pore structure, and its emergence shows that the presence of SAP significantly alters the structure of the cement paste.

For the sample containing SAP-A, $\tau_{3}=1.32 \mathrm{~ns}$ and $\tau_{4}=12.6 \mathrm{~ns}$, with respective intensities of $3.3 \%$ and $1.0 \%$. These lifetimes would correspond to pores having diameters of 0.42 and $1.45 \mathrm{~nm}$ respectively. There is also a redistribution of the weighting of the shorter components $\left(\tau_{1}\right.$ and $\left.\tau_{2}\right)$; however this is not as pronounced as for ordinary Portland cement.

For the sample containing SAP-B, $\tau_{3}=1.62 \mathrm{~ns}$ and $\tau_{4}=14.9 \mathrm{~ns}$, with intensities of $2.5 \%$ and $0.7 \%$ respectively. These lifetimes correspond to pores having diameters of 0.42 and $1.45 \mathrm{~nm}$ respectively. The value of $\tau_{3}$ is comparable to that obtained before heat treatment (which was similar to the o-Ps lifetime in water). This could suggest that the heat treatment did not result in the complete removal of free water.

For both SAP-A and SAP-B the total o-Ps intensity $\left(\mathrm{I}_{3}+\mathrm{I}_{4}\right)$ decreases after heat treatment, signalling a reduction in porosity; however, the reduction is larger for the ordinary Portland cement sample. 


\section{Conclusion}

This study has shown that PALS can be used to monitor the hydration process in cement samples by using the o-Ps intensity as an indicator of the concentration of water-filled pores. In addition, PALS has shown that the addition of a SAP to the cement mixture alters the structure and hydration process of cement. The addition of SAP-A caused the hydration process to progress more gradually compared to OPC; however, the addition of SAP-B gave comparable results to that of OPC.

Both PALS and MIP showed that the presence of SAP-A has a significant effect on the hydration process. In addition, MIP showed that the concentration of pores in the 10-100 $\mathrm{nm}$ range increases with aging for the SAP-A sample.

For the samples with SAP added, heat treatment under evacuation (resulting in the removal of free water) gave rise to an additional long lifetime component and a higher overall o-Ps intensity compared to OPC. This shows that the SAP had modified the pore distribution, replacing some of the regular pore distribution with larger pores formed by saturated polymers.

\section{References}

[1] Bogue, R. H. The Chemistry of Portland Cement. (Reinhold, 1955).

[2] Lea, F. M. The Chemistry of Cement and Concrete. (Arnold, 1970).

[3] Neville, A. M. Properties of Concrete. 4th edn, (Longman, 1995).

[4] Taylor, H. F. W. Cement Chemistry. (Academic, 1990).

[5] Ghosh, S. N. Advances in cement technology (Pergamon, Oxford, 1983).

[6] Gregg, S. J. \& Sing, K. S. W. Adsorption, Surface Area and Porosity. (Academic, 1982).

[7] Jean, Y. C., Mallon, P. E. \& Schrader, D. M. Principles and applications of positron and positronium chemistry (World Scientific, 2003).

[8] Buchholz, F. L. \& Graham, A. T. Modern Superabsorbent Polymer Technology (John Wiley \& Sons, 1997).

[9] Jensen, O. M. \& Hansen, P. F. Water-entrained cement-based materials: I. Principles and theoretical background. Cement and Concrete Research 31, 647-654, (2001).

[10] Jensen, O. M. \& Hansen, P. F. Water-entrained cement-based materials: II. Experimental observations. Cement and Concrete Research 32, 973-978, (2002).

[11] Krause-Rehberg, R. \& Leipner, H. S. Positron Annihilation in Semiconductors. Vol. 127 (Springer, 1999).

[12] Charlton, M. \& Humberston, J. W. Positron Physics. (Cambridge University Press, 2001).

[13] Mogensen, O. E. Spur reaction model of positronium formation. The Journal of Chemical Physics 60 (1974).

[14] Tao, S. J. Positronium Annihilation in Molecular Substances. The Journal of Chemical Physics 56 (1972). 
[15] Eldrup, M., Lightbody, D. \& Sherwood, J. N. The temperature dependence of positron lifetimes in solid pivalic acid. Chemical Physics 63, 51-58, (1981).

[16] Gidley, D. W. et al. Positronium annihilation in mesoporous thin films. Physical Review B 60, R5157 (1999).

[17] Gidley, D. W. et al. Determination of pore-size distribution in lowdielectric thin films. Applied Physics Letters 76 (2000).

[18] Pascual-Izarra, C. et al. Advanced fitting algorithms for analysing positron annihilation lifetime spectra. Nuclear Instruments and Methods in Physics Research Section A: Accelerators, Spectrometers, Detectors and Associated Equipment 603, 456-466, (2009).

[19] Patro, A. P. \& Sen, P. Anomalous parapositronium lifetime in water at 20 degrees C. Journal of Physics C: Solid State Physics 5, 3273 (1972).

[20] Consolati, G. \& Quasso, F. Evolution of porosity in a Portland cement paste studied through positron annihilation lifetime spectroscopy. Radiation Physics and Chemistry 68, 519-521 (2003).

[21] Consolati, G. \& Quasso, F. A positron annihilation study on the hydration of cement pastes. Materials Chemistry and Physics 101, 264-268 (2007).

[22] Consolati, G., Dotelli, G. \& Quasso, F. Positron lifetime spectroscopy as a probe of nanoporosity of cement-based materials. Radiation Physics and Chemistry 58, 727-731 (2000).

[23] Salgueiro, W., Somoza, A., Cabrera, O. \& Consolati, G. Porosity study on free mineral addition cement paste. Cement and Concrete Research 34, 91 97, (2004).

[24] Myllylä, R. \& Karras, M. Positron Annihilation Probing for the Hydratation Rate of Cement Paste. Applied Physics 7, 303-306 (1975).

[25] Sen Wang, P. U. et al. 1H nuclear magnetic resonance characterization of Portland cement: molecular diffusion of water studied by spin relaxation and relaxation time-weighted imaging. Journal of Material Science 33, 3065-3071 (1998). 